Check for updates

Cite this: RSC Adv., 2019, 9, 8569

\title{
Superhydrophobic and superoleophilic graphene aerogel for adsorption of oil pollutants from water
}

\begin{abstract}
Hui Wang, ${ }^{a}$ Chunchun Wang, ${ }^{c}$ Shuai Liu, ${ }^{a}$ Lin Chen ${ }^{\star b}$ and Sudong Yang (D) *a
Three-dimensional graphene based materials with superhydrophobic/superoleophilic attributes are highly desirable for water treatment. The graphene aerogel (GA) was prepared by hydrothermal reaction of the graphene oxide (GO) solution in the presence of dopamine followed by freeze-drying. The subsequent surface modification of GA using fluoroalkylsilane occurred by a vapor-liquid deposition process. The superhydrophobic graphene aerogel (SGA) fabricated from GA exhibits superhydrophobicity and superoleophilicity with the water contact angle of $156.5^{\circ}$ and the oil contact angle of $0^{\circ}$. With this property, SGA could selectively adsorb various types of oils/organic solvents from the oil-water mixture. Moreover, the SGA possesses excellent low bulk density $\left(9.6 \mathrm{mg} \mathrm{cm}^{-3}\right)$, high absorption capacity (110230 fold weight gain), and superior adsorption recyclability. With all these desirable features, the SGA is a promising candidate for oil-polluted water remediation.
\end{abstract}

Received 12th January 2019

Accepted 7th March 2019

DOI: $10.1039 / \mathrm{c} 9 \mathrm{ra00279k}$

rsc.li/rsc-advances synthesized graphene foam for oil adsorption by the thermal reduction of graphene oxide foam. ${ }^{29} \mathrm{Qu}$ and Xie manufactured nitrogen doped graphene framework as the adsorbents of oils and organic solvents. ${ }^{30,31}$ Losic and Gao prepared graphene/ carbon nanotube composite aerogels. ${ }^{32,33}$ Our previous study also reported porous graphene foam with good hydrophobicity and oil adsorbability, which could be used for oil/water separation. ${ }^{34,35}$ Although the above graphene-based monoliths have high adsorption capacity, they have no selectivity for oil and water, decreasing the separation selectivity and efficiency. ${ }^{36,37}$ In order to endow the neat graphene monolith with superhydrophobicity and superoleophilicity so as to further improve its separation efficiency, designing surface structure with high roughness and low surface energy is expected to be a feasible and effective pathway. ${ }^{38-42}$

In this work, the superhydrophobic graphene aerogel (SGA) was prepared by polydopamine (PDA) functionalized GA via hydrothermal method and subsequent hydrophobic modification using fluoroalkylsilane through vapor-liquid deposition. Various techniques were employed to study the morphology and surface properties of the materials. The wetting characteristics of SGA monolith were measured to confirm its superhydrophobicity and superoleophilicity. The oily compounds adsorption and oil-water mixture separation properties of monolith were systematically characterized.

\section{Experiments}

${ }^{a}$ Laboratory of Environmental Science and Technology, The Xinjiang Technica Institute of Physics and Chemistry, Key Laboratory of Functional Materials and Devices for Special Environments, Chinese Academy of Sciences, Urumqi 830011, China. E-mail: yangsd@ms.xjb.ac.cn; Tel: +86-991-6992225

${ }^{b}$ Xinjiang Uygur Autonomous Region Product Quality Supervision and Inspection Institute, Urumqi 830011, China. E-mail: chlin8636@sina.com ${ }^{c}$ SEL BIOCHEM Xinjiang Company Limited, Shihezi 832000, China

\section{Materials}

Powder-like graphite was obtained from Sinopharm Chemical Reagent Co. (China). Dopamine hydrochloride (DA) and $1 H, 1 H, 2 H, 2 H$-perfluorooctyltriethoxysilane (PFOES) were 
obtained from Sigma-Aldrich. All chemicals were analytical grade and used as received without further purification.

\section{Preparation of aerogel}

GO was prepared from graphite powder through a modified Hummer's method as reported before. ${ }^{43}$ In a typical experiment, $17.0 \mathrm{mg}$ of GO was ultrasonically dispersed in $5.0 \mathrm{~mL}$ water for $4 \mathrm{~h}$. Then $0.31 \mathrm{~mL}$ of DA solution $\left(16.0 \mathrm{mg} \mathrm{mL}^{-1}\right)$ was added into the GO dispersion and stirred for $10 \mathrm{~min}$. The mixture was then transferred into a Teflon-lined stainless steel autoclave and hydrothermally processed at $120{ }^{\circ} \mathrm{C}$ for $12 \mathrm{~h}$. Finally, the treated hydrogel sample was freeze-dried to get GA.

The obtained aerogel was placed in a glass vessel filled with an ethanolic solution of (PFOES) ( $2 \mathrm{wt} \%$ ) with no direct contact between the liquid and the aerogel. Subsequently, the glass vessel was sealed and heated at $70^{\circ} \mathrm{C}$ for $8 \mathrm{~h}$. The SGA resulted after thoroughly drying at $100{ }^{\circ} \mathrm{C}$ for $1 \mathrm{~h}$.

\section{Adsorption capacity of aerogel}

Different organics, such as crude oil, gasoline, diesel, engine oil, peanut oil, $n$-hexane, octane, hexadecane, chloroform, and 1,2dichlorobenzene were used to evaluate oil adsorption capacity of aerogel. Typically, the aerogel was immersed in the oils for 10 minutes and then picked up using tweezers. After removing the redundant oil on the surface, the aerogel was measured as soon as possible on the mass balance. The oil adsorption capacity was obtained by the weight imparity before and after the immersion.

\section{Characterization}

The morphology of aerogel was observed by scanning electron microscopy (SEM, Supra 55VP, Zeiss, Germany). Elemental analysis was carried out by energy dispersive spectroscopy (EDS), and the EDS spectra were obtained through a Zeiss Supra 55VP SEM instrument equipped with an EDS detector. Surface functionalities and elemental composition of aerogel were studied using an X-ray photoelectron spectroscopy (XPS, EscaLab 250Xi, Thermo Scientific, US). Functional groups in sample were analyzed through a Fourier-transform infrared spectrometer (FT-IR, NICOLET 5700). The static contact angle of aerogel against water was measured on a goniometer (XG-CAMA, Shanghai Xuanyichuangxi, China) at ambient temperature. The optical images of the sample were acquired using a digital camera (D7000, Nikon, Japan).

\section{Results and discussion}

The synthetic routes of SGA are illustrated in Fig. 1. First, the PDA functionalized graphene hydrogel was prepared by hydrothermal reduction of the mixtures of GO and dopamine. Second, the graphene hydrogel was by freeze-drying to produce GA. Third, the low-energy PFOES layer coated on the GA surface through the reaction of the hydroxyl groups in GA with the alkoxysilane group of PFOES. Finally, the resulting SGA was obtained by using the vapor-liquid deposition method. Different from the frequently used SGA prepared solutionprocessed coating approaches, vapor-liquid deposition avoid the unmanageable solvent removal process and can maintain the stable shape, initial monolithic volume and structure of the materials. Importantly, the surface of the graphene based material can be modified according to the need of oil-water separation. As a result, a piece of SGA could effortlessly stand on the top of foliage without deforming the supporter at all (Fig. 1). The calculated density of aerogel based on the weight and dimension of sample was $9.6 \mathrm{mg} \mathrm{cm}^{-3}$, belonged to the range of ultra-low density material.

The morphology of the surface of GA and SGA was examined by SEM. SEM images of the surface of GA in different magnifications are shown in Fig. 2a and c. As revealed by SEM images of the GA (Fig. 2a), the curly graphene nanosheets were randomly cross-linked to form 3D interconnected hierarchical porous network with pore size distributions in the scope of submicrometers to ten micrometers. The self-assembly of graphene nanosheets into the $3 \mathrm{D}$ structures could be attributed to the partial overlapping or coalescence of the flexible reduced GO nanosheets via noncovalent interactions, such as hydrogen bonding, and $\pi-\pi$ interactions. ${ }^{\mathbf{4 4}, 45}$ The surface of the graphene was smooth at the magnified scale (Fig. 2c). The smooth surface of GA indicated that PDA was uniformly coated on the basal planes of graphene, which might be due to the strong affinity between the dopamine aromatic rings and graphene nanosheets. ${ }^{46}$ After the in situ vapor-liquid deposition process, a similar porous morphology with interconnected frameworks was observed for SGA (Fig. 2b). However, a compact coating with a random distribution of many nanoscale granules was observed on the graphene surface (Fig. 2d), which showed that the accomplishment of covalent interaction on the surface of SGA. The nanoparticle morphology provided the nanoscale roughness to complement the microscale roughness inherent in the graphene aerogel. Therefore, the surface microscale and nanoscale roughness was an essential necessity to realize the superhydrophobicity of SGA. Energy dispersive spectroscopy (EDS) analysis was used to further investigate the chemical composition of aerogels. As shown in Fig. 2e, only peaks of $\mathrm{C}, \mathrm{O}$, and $\mathrm{N}$ were detected on the GA, and no other impurities could be observed. After the deposition process and hydrophobic modification, new peaks of $\mathrm{F}$ and $\mathrm{Si}$ could be observed besides

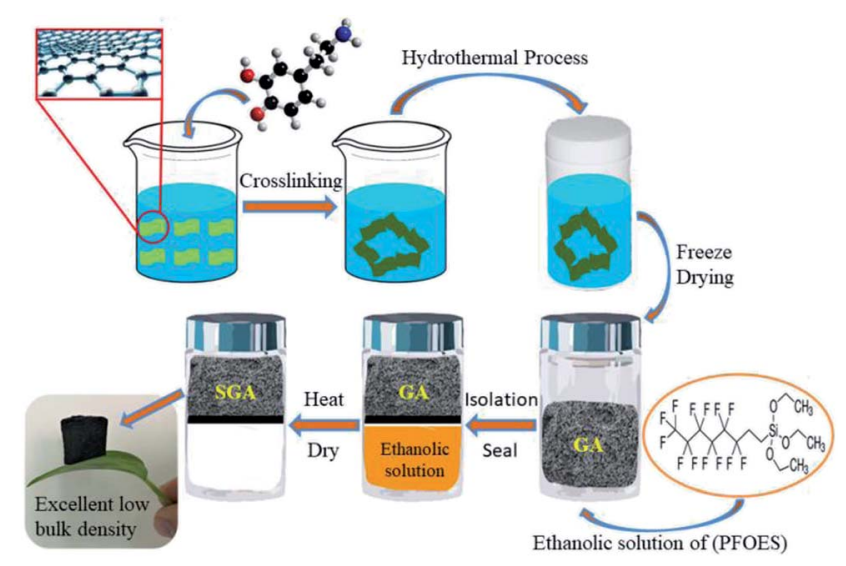

Fig. 1 The fabrication process and the digital image of SGA. 


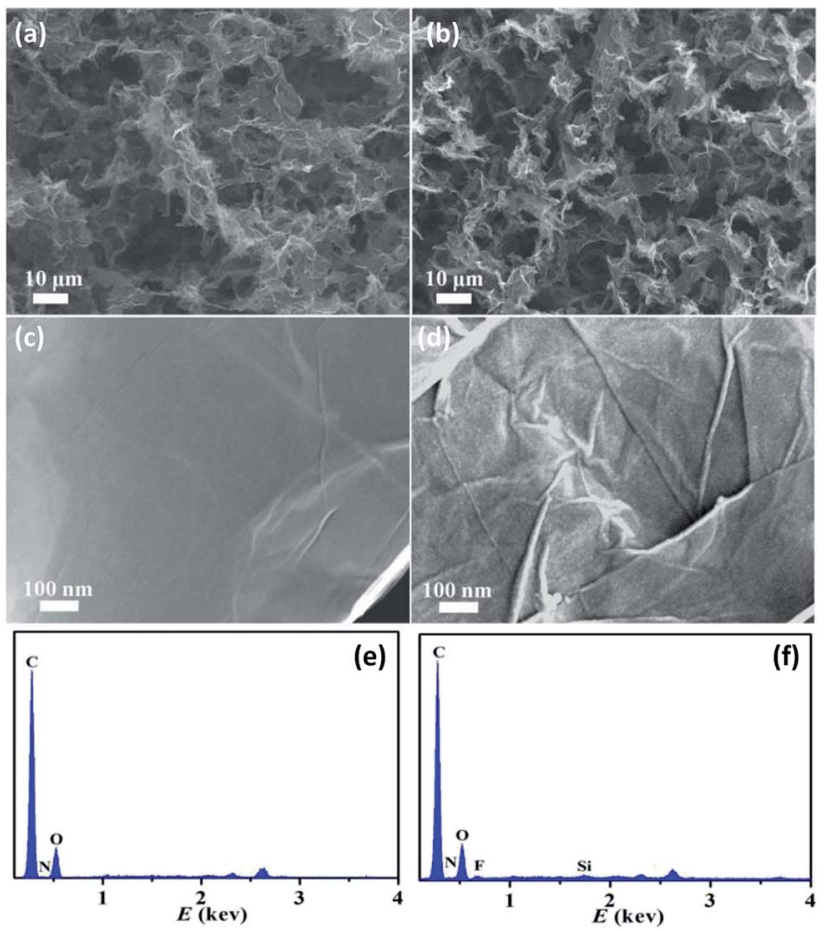

Fig. 2 Low- and high-magnification SEM images and corresponding EDS analysis of ( $a, c$ and e) GA and (b, $d$ and f) SGA.

C, O, and N for the SGA (Fig. 2f). These results show that F and Si element is successful introduction overall the surface of SGA. The 3D porous and hydrophobic structures of SGA are highly desirable for oil and organic solvent adsorption.

To further verify the formation mechanism of the superhydrophobic surface, XPS measurements were used to compare the chemical composition of the GO, GA, and SGA. The peaks of C1s and O1s were observed in GO, while a new peak of N1s emerged in GA, which should arise from polydopamine on the graphene hydrogel during the process of preparing (Fig. 3a). After the modification process, the XPS spectrum of SGA (Fig. 3a) had fluorine component originating from SGA, indicating that the covalent functionalization of GA by PFOES successfully occurred. This result was consistent with the results of the EDS analysis. The detailed deconvolutions of the C1s spectra for GO, GA, and SGA were investigated as shown in Fig. 3b. Deconvolution of the C1s signal in GO sample showed a strong peak at high binding energy for the heavily oxygenated carbon species. This result was consistent with the results of the previous GO. ${ }^{35}$ After hydrothermal reduction, the intensity of the peak was decreased obviously for the heavily oxygenated carbon species, and the peak related to $\mathrm{C}=\mathrm{C} / \mathrm{C}-\mathrm{C}(\sim 284.6 \mathrm{eV})$ became dominant (Fig. 3b). Furthermore, deconvolutions of the C1s spectrum for SGA appeared new peak associated with the $\mathrm{C}-\mathrm{F}$ covalent bonds at about $291.9 \mathrm{eV}$ corresponding to $\mathrm{C}-\mathrm{F}$ bonding (Fig. 3b). ${ }^{47}$ The new Si2p (102.3 eV) (Fig. 3c) and F1s $(689.2 \mathrm{eV})$ (Fig. 3d) peak in the XPS spectrum of the SGA confirmed the successful binding of fluoroalkylsilane on the surface of the as prepared SGA. The atomic ratios of carbon, oxygen, nitrogen, silicon, and fluorine measured by XPS in
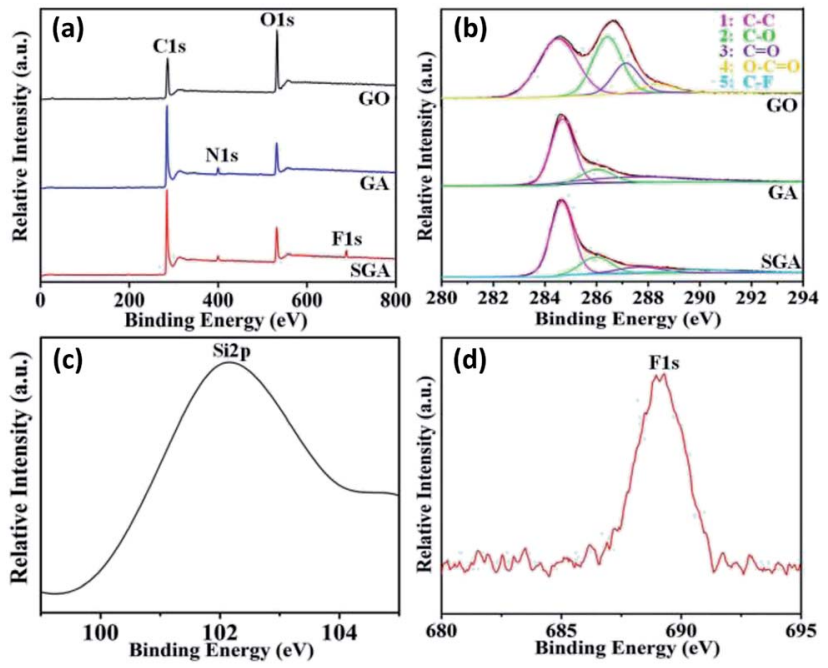

Fig. 3 XPS results. (a) XPS wide-scan spectra and (b) C1s high-resolution spectra of the GO, GA, and SGA. (c) Si2p and (d) F1s highresolution spectra of SGA.

different materials were summarized in Table 1. After reduction, the content of carbon increased gradually to $81.07 \%$ while the oxygen content decreased gradually. This result shows that the majority of oxygen-containing groups are removed and some of them may be from PDA (Fig. 3b). GA contained 1.62\% of $\mathrm{N}$ originating from the PDA, indicating that the PDA successfully deposited on surface of the GA. Additionally, after the hydrophobic modification process, the new element of silicon and fluorine content in the SGA sample was $0.72 \%$ and $2.12 \%$, respectively. The above results indicate fluorine and silanes are chemically bound on the SGA surface by the hydrolysis/condensation reaction.

The FT-IR spectra of GA and SGA were shown in Fig. 4. For the spectrum of the GA, the peak appeared at $1445 \mathrm{~cm}^{-1}(\mathrm{~N}-\mathrm{H}$ of amide group shearing vibration) and $1677 \mathrm{~cm}^{-1}(\mathrm{~N}-\mathrm{H}$ in-plane stretching vibration), indicating the presence of amine groups after polydopamine-mediated assembly. After surface functionalization with PFOES, new bands at 1162, 1262, and $1385 \mathrm{~cm}^{-1}$ were rocking vibration peaks of $\mathrm{C}-\mathrm{F}$ bond, and two bands at 2917 and $2961 \mathrm{~cm}^{-1}$ appeared, showing the stretching of the $-\mathrm{CH}_{2}$ groups from the alkyl chains assigning to silane moieties of PFOES-GAs. ${ }^{48}$ Besides, the bands at 1065 and $1109 \mathrm{~cm}^{-1}$ were attributed to the $\mathrm{Si}-\mathrm{O}-\mathrm{Si}$ and $\mathrm{Si}-\mathrm{O}-\mathrm{C}$ bonds, indicating the successful chemical functionalization of GA by PFOES. ${ }^{49}$

The wetting behavior of the SGA is shown in Fig. 5. The dynamic wettability of water and oil on the surface was investigated through the high-speed camera system to record the liquid droplet permeating process. For the water droplet

Table 1 Atomic ratio of GO, GA and SGA

\begin{tabular}{llllll}
\hline Sample & C (\%) & O (\%) & N (\%) & Si (\%) & F (\%) \\
\hline GO & 66.67 & 33.33 & - & - & - \\
GA & 81.07 & 17.31 & 1.62 & - & - \\
SGA & 77.34 & 18.12 & 1.7 & 0.72 & 2.12
\end{tabular}




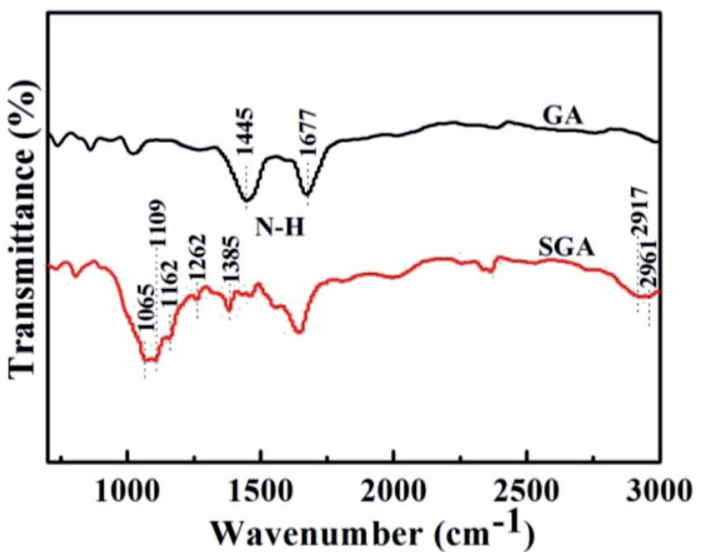

Fig. 4 FT-IR spectra of GA and SGA.

adhesion performance, a water droplet was driven to completely contact with the surface of SGA and then lifted it up. From the corresponding photographs of the water droplet (Fig. 5a), no deformation was observed when water droplet left the surface of aerogel. The result confirms the SGA has extremely low water adhesion. Conversely, when oily liquid droplet contacted with SGA, it continuously spread and permeated on the material surface with the contact angles of $0^{\circ}$ (Fig. 5b). The SGA can adsorb the hexadecane droplet within $5 \mathrm{~s}$. The SGA behaves as a superior oil-adhesion property. The SGA exhibit the superhydrophobicity with a water contact angle of $156.5^{\circ}$, while the oil drop is adsorbed completely by the SGA and no contact angle can be found. Solid surface energy was 76.82 and $0.96 \mathrm{mN} \mathrm{m}^{-1}$ for the GA and SGA, respectively, which was calculated by Owen's two liquid methods. The lower surface energy of the SGA is crucial for get superoleophilic surface in air. The superhydrophobic and superoleophilic wettability of SGA avoids direct contact with water and ensures fast oil permeation and moving during oil-water separation process.

Owing to the above-mentioned superhydrophobicity, hierarchical structure, and ultra-low density, the aerogels are considered as a promising material for highly efficient oil-water separation and organic solvents adsorption. As

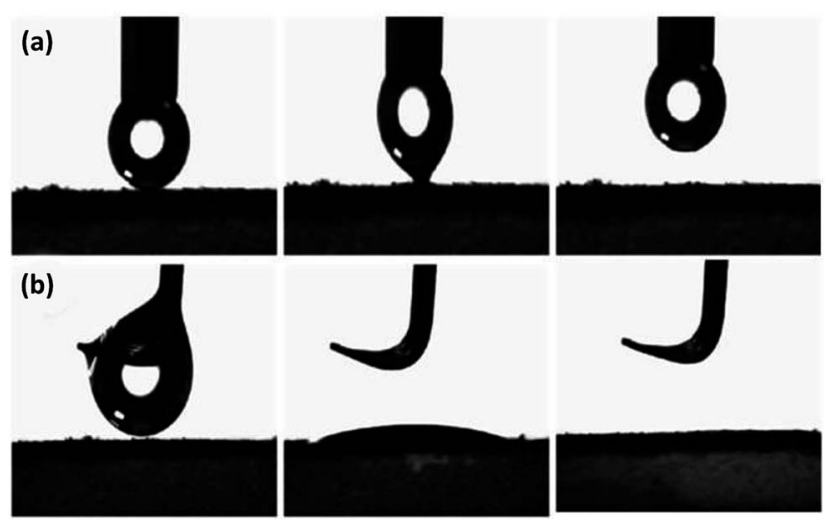

Fig. 5 Wetting behavior of (a) water and (b) hexadecane droplet on SGA surface. a demonstration, the light oil atop water surface (Fig. 6a) and heavy oil underwater (Fig. 6b) were used as an example to show the adsorption process of SGA. Fig. 6a showed the adsorption performance of SGA to light oil atop water. The SGA could immediately adsorb the hexadecane layer (dyed with oil red) around it and leave transparent regions on water surface when a piece of it contacted with the hexadecane on water. The hexadecane was completely absorbed by SGA within a few seconds because the SGA had a rapid adsorption process for oils and organic solvents on water. Fig. $6 \mathrm{~b}$ demonstrated that SGA could quickly adsorb heavy oil underwater as well. The droplet of chloroform was immediately sucked into the aerogel once the SGA was immersed into water and started to contact with chloroform. There was no detectable water in the adsorbed aerogel materials. The above results demonstrate the SGA has excellent adsorption selectivity for immiscible oils-water. The adsorption performance of SGA was further evaluated through a series of adsorption experiments for various organic solvents and oils. Compared with GA, SGA has a high adsorption capacity (Fig. 6c). It can be seen that adsorption capacity of SGA in the range of 110-230 times higher than its own weight for various oils and organic solvents (Fig. 6c). The capacities of SGA for all oily liquids are over $100.0 \mathrm{~g} \mathrm{~g}^{-1}$. The SGA showed high adsorption capacity for various oils and organic solvents, which were much higher than most previous activated carbon and polymers, ${ }^{50,51}$ and superhydrophobic graphene-based aerogels reported in the previous literatures. ${ }^{26,52}$ Furthermore, similar to the previously reported, ${ }^{33}$ the adsorption capacity of SGA was
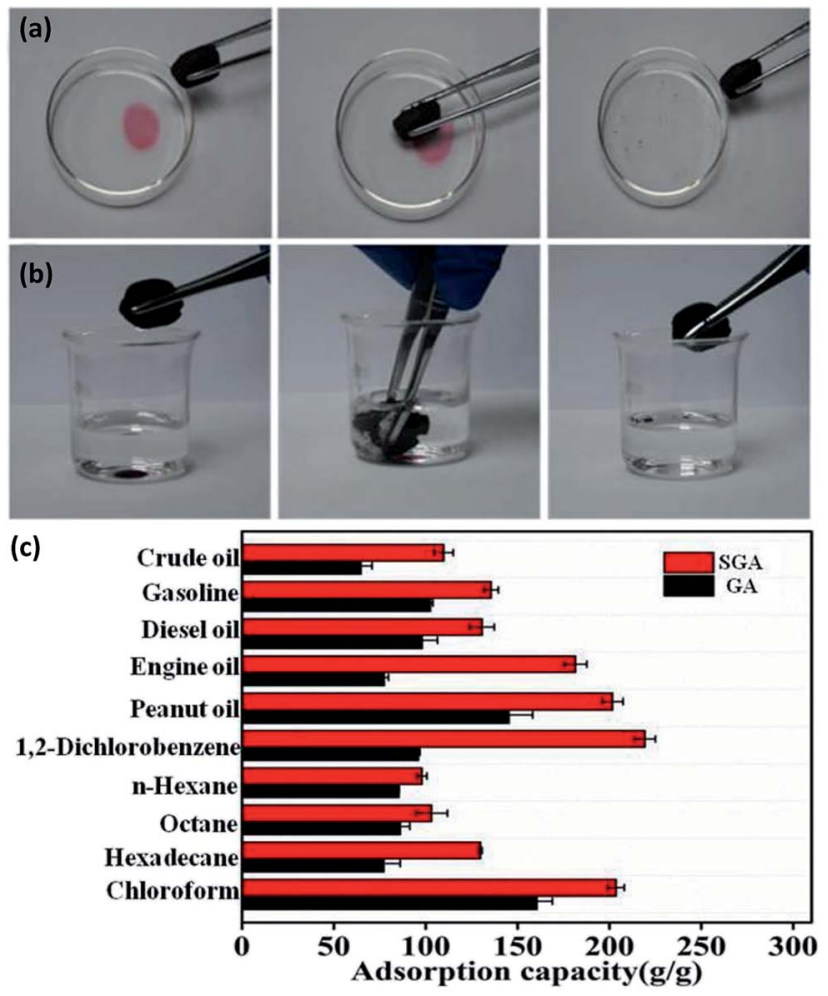

Fig. 6 Oil adsorption performance of different aerogels (a) illustration showing the adsorption process of SGA for hexadecane and (b) chloroform; (c) adsorption capacity of SGA and GA for various organic liquids. 


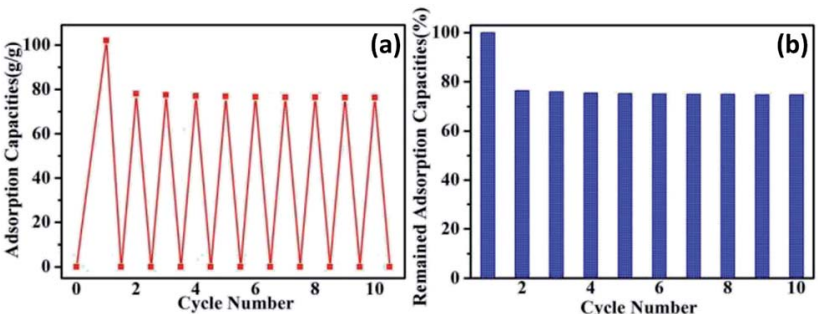

Fig. 7 Recyclability of SGA for oil adsorption ((a) adsorption recyclability of aerogel over ten times cycles; (b) remained adsorption capacity of sample).

approximately proportional to the density of the various solvents. Combined homogeneous internal structure with low surface energy, the adsorption capacity of SGA toward oil and other organic chemicals is greatly improved.

The recyclability and recoverability are important property for an ideal adsorption material in the actual application of oil cleaning. As shown in Fig. 7, octane was used as an example to evaluate the recycling potential and recovering of SGA for the oils and organic solvents through a simple sorption-drying cycle. As a result, only a small decline for the adsorption capacity of SGA was observed after ten times adsorptiondesorption cycles test. The adsorption capacity was still higher than $70 \mathrm{~g} \mathrm{~g}^{-1}$ for the used common solvent. This result highlights the excellent recyclability of SGA for oil-adsorption by taking this simple sorption-drying method over ten repeat times. Notably, a slight drop for oil adsorption capacity may be attributed to the residual oil entrained in the pores of the aerogels. ${ }^{53}$ These results indicate the good recyclability and recoverability of SGA by a simple sorption-drying cycle method.

\section{Conclusion}

In summary, SGA with superhydrophobic and oleophilic properties was prepared from PDA functionalized graphene aerogel via hydrothermal method and subsequent hydrophobic modification using fluoroalkylsilane through vapor-liquid deposition. The modification not only introduces nanoscale roughness on the surface of the graphene, but also decreases the surface tension. The resulting SGA showed a high water contact angle of $156.5^{\circ}$ and low water adhesion property. Furthermore, the SGA can selective removal of organic pollutants from water and adsorb a broad variety of oil liquids with enormous adsorption capacities. The superhydrophobic GA is very stable in oils and can be used repeatedly for oil/water separation.

\section{Conflicts of interest}

There are no conflicts to declare.

\section{Acknowledgements}

This project was supported by Natural Science Foundation of Xinjiang Uygur Autonomous Region, China (Project No. 2017D01A74).

\section{Notes and references}

1 I. B. Ivshina, M. S. Kuyukina, A. V. Krivoruchko, A. A. Elkin, S. O. Makarov, C. J. Cunningham, T. A. Peshkur, R. M. Atlas and J. C. Philp, Environ. Sci.: Processes Impacts, 2015, 17, 1201-1219.

2 J. Ge, H. Y. Zhao, H. W. Zhu, J. Huang, L. A. Shi and S. H. Yu, Adv. Mater., 2016, 28, 10459-10490.

3 Y. Q. Wang, Y. Feng and J. F. Yao, J. Colloid Interface Sci., 2019, 533, 182-189.

4 M. A. Riaz, P. Hadi, I. H. Abidi, A. Tyagi, X. W. Ou and Z. T. Luo, RSC Adv., 2017, 7, 29722-29731.

5 W. W. Zhan, S. R. Yu, L. Gao, F. Wang, X. Fu, G. Sui and X. P. Yang, ACS Appl. Mater. Interfaces, 2018, 10, 1093-1103.

6 J. Saleem, M. A. Riaz and G. McKay, J. Hazard. Mater., 2018, 341, 424-437.

7 F. Z. Chen, Y. Lu, X. Liu, J. L. Song, G. J. He, M. K. Tiwari, C. J. Carmalt and I. P. Parkin, Adv. Funct. Mater., 2017, 27, 1702926.

8 W. C. Wan, Y. H. Lin, A. Prakash and Y. Zhou, J. Mater. Chem. A, 2016, 4, 18687-18705.

9 C. H. Lee, B. Tiwari, D. Y. Zhang and Y. K. Yap, Environ. Sci.: Nano, 2017, 4, 514-525.

10 E. B. Kujawinski, M. C. Kido Soule, D. L. Valentine, A. K. Boysen, K. Longnecker and M. C. Redmond, Environ. Sci. Technol., 2011, 45, 1298-1306.

11 C. Nam, H. X. Li, G. Zhang, L. R. Lutz, B. Nazari, R. H. Colby and T. C. M. Chung, ACS Sustainable Chem. Eng., 2018, 6, 12036-12045.

12 J. Ge, Y. D. Ye, H. B. Yao, X. Zhu, X. Wang, L. Wu, J. L. Wang, H. Ding, N. Yong, L. H. He and S. Yu, Angew. Chem., 2014, 126, 3686-3690.

13 J. Aurell and B. K. Gullett, Environ. Sci. Technol., 2010, 44, 9431-9437.

14 R. A. Gardner, R. Kinkade, C. J. Wang and O. Phanstiel, J. Org. Chem., 2004, 69, 3530-3537.

15 Z. Z. Li, B. Wang, X. M. Qin, Y. K. Wang, C. T. Liu, Q. Shao, N. Wang, J. X. Zhang, Z. K. Wang, C. Y. Shen and Z. H. Guo, ACS Sustainable Chem. Eng., 2018, 6, 13747-13755.

16 X. Gui, J. Wei, K. Wang, A. Cao, H. Zhu, Y. Jia, Q. Shu and D. Wu, Adv. Mater., 2010, 22, 617-621.

17 D. D. Nguyen, N. H. Tai, S. B. Lee and W. S. Kuo, Energy Environ. Sci., 2012, 5, 7908-7912.

18 H. C. Bi, Z. Y. Yin, X. H. Cao, X. Xie, C. L. Tan, X. Huang, B. Chen, F. T. Chen, Q. L. Yang, X. Y. Bu, X. H. Lu, L. T. Sun and H. Zhang, Adv. Mater., 2013, 25, 5916-5921.

19 Z. Y. Wu, C. Li, H. W. Liang, Y. N. Zhang, X. Wang, J. F. Chen and S. H. Yu, Sci. Rep., 2015, 4, 4079.

20 O. S. H. Santos, M. C. da Silva, V. R. Silva, W. N. Mussel and M. I. Yoshida, J. Hazard. Mater., 2017, 324, 406-413.

21 Y. T. Liu, J. W. Sun, J. G. Yuan, S. Wang, Y. Ding, Y. M. Wu and C. H. Gao, Inorg. Chem. Front., 2018, 5, 1894-1901.

22 J. F. Li, Y. H. Chen, J. Gao, Z. C. Zuo, Y. J. Li, H. B. Liu and Y. L. Li, ACS Appl. Mater. Interfaces, 2019, 11, 2591-2598.

23 T. A. Tabish, F. A. Memon, D. E. Gomez, D. W. Horsell and S. W. Zhang, Sci. Rep., 2018, 8, 1817. 
24 A. A. Nikkhah, H. Zilouei, A. Asadinezhad and A. Keshavarz, Chem. Eng. J., 2015, 262, 278-285.

25 S. Wang, Y. Zhang, N. Abidi and L. Cabrales, Langmuir, 2009, 25, 11078.

26 H. C. Bi, X. Xie, K. B. Yin, Y. L. Zhou, S. Wan, L. B. He, F. Xu, F. Banhart, L. T. Sun and R. S. Ruoff, Adv. Funct. Mater., 2012, 22, 4421-4425.

27 J. L. Wang, Z. X. Shi, J. C. Fan, Y. Ge, J. Yin and G. X. Hu, J. Mater. Chem., 2012, 22, 22459-22466.

28 J. Li, J. G. Li, H. Meng, S. Y. Xie, B. W. Zhang, L. F. Li, H. J. Ma, J. Y. Zhang and M. Yu, J. Mater. Chem. A, 2014, 2, 2934-2941.

29 Y. He, Y. Liu, T. Wu, J. K. Ma, X. R. Wang, Q. J. Gong, W. N. Kong, F. B. Xing, Y. Liu and J. P. Gao, J. Hazard. Mater., 2013, 260, 796-805.

30 X. H. Song, L. P. Lin, M. C. Rong, Y. R. Wang, Z. X. Xie and X. Chen, Carbon, 2014, 80, 174-182.

31 Y. Zhao, C. G. Hu, Y. Hu, H. H. Cheng, G. Q. Shi and L. T. Qu, Angew. Chem., Int. Ed., 2012, 51, 11371-11375.

32 S. Kabiri, D. N. H. Tran, T. Altalhi and D. S. Losic, Carbon, 2014, 80, 523-533.

33 H. Y. Sun, Z. Xu and C. Gao, Adv. Mater., 2013, 25, 2554-2560.

34 S. D. Yang, L. Chen, L. Mu and P. C. Ma, J. Colloid Interface Sci., 2014, 430, 337-344.

35 S. D. Yang, L. Chen, L. Mu, B. Hao, J. T. Chen and P. C. Ma, RSC Adv., 2016, 6, 4889-4898.

36 Q. Zhu, Q. M. Pan and F. T. Liu, J. Phys. Chem. C, 2011, 115, 17464-17470.

37 R. Li, C. B. Chen, J. Li, L. M. Xu, G. Y. Xiao and D. Y. Yan, J. Mater. Chem. A, 2014, 2, 3057-3064.

38 X. C. Dong, J. Chen, Y. W. Ma, J. Wang, M. B. Chan-Park, X. Liu, L. Wang, W. Huang and P. Chen, Chem. Commun., 2012, 48, 10660-10662.
39 H. C. Bi, X. Xie, K. B. Yin, S. Wan, R. S. Ruoff and L. T. Sun, J. Mater. Chem. A, 2014, 2, 1652-1656.

40 E. Singh, Z. P. Chen, F. Houshmand, W. C. Ren, Y. Peles, H. M. Cheng and N. Koratkar, Small, 2013, 9, 75-80.

41 L. M. Xu, G. Y. Xiao, C. B. Chen, R. Li, Y. Y. Mai, G. M. Sun and D. Y. Yan, J. Mater. Chem. A, 2015, 3, 7498-7504.

42 C. C. Wang, S. D. Yang, Q. Ma, X. Jia and P. C. Ma, Carbon, 2017, 118, 765-771.

43 S. D. Yang, C. M. Shen, Y. Y. Liang, H. Tong, W. He, X. Z. Shi, X. G. Zhang and H. J. Gao, Nanoscale, 2011, 3, 3277-3284.

44 H. Bai, C. Li, X. L. Wang and G. Q. Shi, J. Phys. Chem. C, 2011, 115, 5545-5551.

45 H. C. Gao, F. Xiao, C. B. Ching and H. W. Duan, ACS Appl. Mater. Interfaces, 2012, 4, 2801-2810.

46 S. M. Kang, S. Park, D. Kim, S. Y. Park, R. S. Ruoff and H. Lee, Adv. Funct. Mater., 2011, 21, 108-112.

47 J. T. Robinson, J. S. Burgess, C. E. Junkermeier, S. C. Badescu, T. L. Reinecke, F. K. Perkins, M. K. Zalalutdniov, J. W. Baldwin, J. C. Culbertson, P. E. Sheehan and E. S. Snow, Nano Lett., 2010, 10, 30013005.

48 H. F. Yang, F. H. Li, C. S. Shan, D. X. Han, Q. X. Zhang, L. Niu and A. Ivaska, J. Mater. Chem., 2009, 19, 4632-4638.

49 W. S. Ma, J. Li, B. J. Deng and X. S. Zhao, J. Mater. Sci., 2013, 48, 156-161.

50 M. A. Lillo-Rodenas, D. Cazorla-Amoros and A. LinaresSolano, Carbon, 2005, 43, 1758-1767.

51 A. Li, H. X. Sun, D. Z. Tan, W. J. Fan, S. H. Wen, X. J. Qing, G. X. Li, S. Y. Li and W. Q. Deng, Energy Environ. Sci., 2011, 4, 2062-2065.

52 T. Wu, M. G. Chen, L. Zhang, X. Y. Xu, Y. Liu, J. Yan, W. Wang and J. P. Gao, J. Mater. Chem. A, 2013, 1, 7612-7621.

53 M. Khosravi and S. Azizian, ACS Appl. Mater. Interfaces, 2015, 7, 25326-25333. 